\title{
Oclusão da Artéria Umbilical como Opção Terapêutica em Gestações com Gêmeo Acárdio
}

\author{
Umbilical Artery Occlusion as a Therapeutic Option in Pregnancy with Acardiac Twin \\ Júlio César de Faria Couto, Juliana Moysés Leite
}

\begin{abstract}
RESUM0
Gestação gemelar com gêmeo acárdio é um evento raro que se caracteriza pela presença de uma anastomose placentária entre o gêmeo doador, morfologicamente normal, e o gêmeo acárdio, levando à perfusão contra-corrente de um dos gêmeos. As principais complicações encontramse associadas à prematuridade e insuficiência cardiaca do gêmeo normal secundária à sobrecarga circulatória. Várias modalidades terapêuticas têm sido propostas, mas não há consenso em relação aos resultados observados. A oclusão intra-fetal da artéria umbilical do gêmeo acárdio tem se mostrado um método de baixo custo, fácil execução e alta eficácia no controle da sobrecarga circulatória. Apresentamos dois casos com ablação intra-fetal e boa evolução. Uma gestação trigemelar na qual se realizou a ablação intra-fetal com 29 semanas, evoluindo com parto prematuro e nascimento de um recém-nascido em boas condições. Uma gestação gemelar cuja ablação foi realizada com 31 semanas e que evoluiu com rotura prematura de membranas e parto prematuro com nascimento de uma criança saudável.
\end{abstract}

PALAVRAS-CHAVE: Gemelaridade. Transfusão arterial reversa. Acárdio.

\section{Introdução}

Gestação gemelar com gêmeo acárdio é evento raro, de alta morbimortalidade, com incidência estimada de 1/35.000 partos, 1/100 gestações gemelares monozigóticas e 1/30 gestações trigemelares monozigóticas ${ }^{1}$. Ocorre devido à presença de anastomoses placentárias artério-arteriais e veno-venosas levando à perfusão retrógrada do gêmeo acárdio por sangue proveniente do gêmeo normal ${ }^{2}$. Essa condição hemodinâmica peculiar tem sido denominada seqüência TRAP (twin reverse artery perfusion). O gêmeo normal comumente apresenta insuficiência cardíaca secundária à sobrecarga circulatória e ao rápido crescimento do gêmeo acárdio. Além disso, há risco de parto prematuro em conseqüência do poliidrâmnio ${ }^{3}$.

Em virtude das complicações cardiovascu-

Santa Casa de Belo Horizonte - MG

Correspondência:

Júlio César Faria Couto

Rua Nelson Soares de Faria, 366/101 - Cidade Nova

31170-030 - Belo Horizonte - MG

Telefone: (31) 8853-1517 - Fax:(31)3261-6013

e-mail: juliocouto2001@yahoo.com.br lares potencialmente letais ao gêmeo sadio, várias modalidades terapêuticas têm sido propostas, com ênfase aos procedimentos invasivos que visam interromper a perfusão do gêmeo acárdio ${ }^{4}$. As opções terapêuticas com melhores resultados na atualidade consistem na oclusão do cordão e na ablação intrafetal dos vasos do gêmeo acárdio ${ }^{2}$. Nesse relato apresentamos a evolução de uma gestação gemelar e outra trigemelar com gêmeo acárdio após ablação dos vasos fetais com substâncias esclerosantes, além de revisão e discussão da terapia pré-natal.

\section{Caso 1}

Paciente de 19 anos, $\mathrm{G}_{2} \mathrm{P}_{1} \mathrm{~A}_{0}$, encaminhada ao serviço de Medicina Fetal da Santa Casa de Belo Horizonte em maio de 1998, na 24ª semana de gestação, para a realização de ultra-sonografia de rotina que revelou gestação trigemelar, monocoriônica e triamniótica espontânea. Os gêmeos 1 e 3 tinham morfologia normal, sendo que o gêmeo 1 apresentava poliidrâmnio (ILA $=38 \mathrm{~cm}$ ). O gêmeo 2 apresentava-se com pólo cefálico, membros superiores 
e câmaras cardíacas ausentes, tórax rudimentar, abdome apresentando estruturas com diferenciação incompleta sugestivas de alças intestinais e artéria umbilical única, compatível com gêmeo acárdio (Figura 1). Foi prescrito digoxina $(0,25 \mathrm{mg}$ bid durante três dias, seguido por 0,25 mg diariamente). Após duas semanas, devido ao aumento do líquido amniótico no gêmeo 1 e aparecimento de contrações uterinas, foram prescritos indometacina e corticoterapia. Na 29a semana de gestação, o gêmeo 1 evoluiu com cardiomegalia, derrame pericárdico e hepatomegalia. O Doppler venoso demonstrou aumento no índice de pulsatilidade da veia cava inferior, ducto venoso e veia hepática direita. O gêmeo 3 apresentava evolução normal. Essas alterações eram compativeis com descompensação hemodinâmica do gêmeo 1 e intervenção com objetivo de ocluir o suprimento sangüíneo para o gêmeo acárdio foi considerada a melhor opção. Após consentimento do casal foi realizada embolização com oleato de monoetanolamina e glicose hipertônica na proporção de $2: 1$, injetados bilateralmente na porção intra-abdominal da artéria umbilical do gêmeo acárdio, resultando em imediata interrupção do fluxo arterial sem repercussão cardiovascular para os demais gêmeos. Novo ultra-som realizado após 48 horas mostrou que o fluxo sangüíneo havia se restabelecido, associado a deterioração hemodinâmica do gêmeo 1 , com surgimento de pulsatilidade na veia umbilical. Na $30^{\mathrm{a}}$ semana de gestação, a paciente apresentou febre, sinais laboratoriais de infecção e trabalho de parto prematuro, evoluindo para cesariana. $\mathrm{O}$ recém-nascido 1, que correspondia ao gêmeo bomba, era do sexo feminino, pesou $1.250 \mathrm{~g}$ e evoluiu para óbito após 30 minutos. O recém-nascido 3, feminino, pesou 1.150 g e apresentou parada cardiorespiratória após a extração, sendo prontamente reanimado e conduzido ao CTI. Exames laboratoriais mostraram anemia grave associada a insuficiência cardíaca, compensada após sucessivas transfusões sangüineas. Sua evolução pós-natal foi complicada por sepse, doença da membrana hialina grave e icterícia. Evoluiu satisfatoriamente recebendo alta hospitalar após 40 dias. O recém-nascido 2 correspondia ao gêmeo acárdio, cujo estudo anatomopatológico confirmou o diagnóstico de gêmeo acárdio-acéfalo, feminino, pesando $1.050 \mathrm{~g}$. O cariótipo dos recém-nascidos não apresentou alterações $(46 X X)$. A mãe evoluiu com insuficiência respiratória grave durante a cesariana, sendo encaminhada ao CTI com pneumonia bilateral, SARA e sepse. Apresentou boa evolução, recebendo alta hospitalar após 11 dias. Aos 4 anos de idade, a criança não apresenta qualquer tipo de seqüela de desenvolvimento.

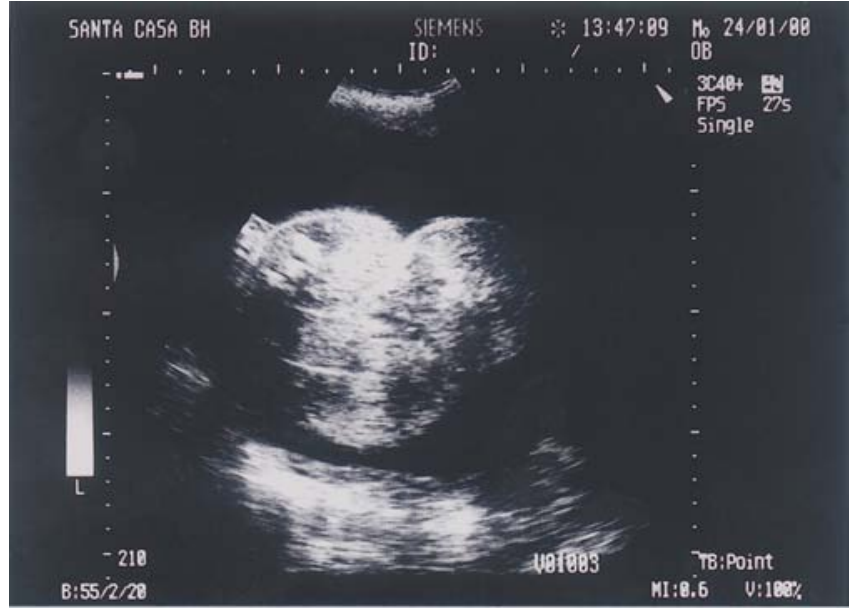

Figura 1 - Aspecto ultra-sonográfico do gêmeo acárdio evidenciando extenso edema subcutâneo.

\section{Caso 2}

Paciente de 29 anos, $\mathrm{G}_{2} \mathrm{P}_{0} \mathrm{~A}_{1}$, encaminhada ao serviço de Medicina Fetal da Santa Casa de Belo Horizonte em janeiro de 2000 , na $27^{\text {a }}$ semana, com diagnóstico de gestação gemelar espontânea, monocoriônica-diamniótica, associada a poliidrâmnio. A ultra-sonografia revelou gêmeo 1 morfologicamente normal, com biometria compatível com a amenorréia e poliidrâmnio (ILA $=51$ $\mathrm{cm})$. O gêmeo 2 apresentava-se com pólo cefálico, membros superiores e câmaras cardíacas ausentes. O diagnóstico foi compativel com gestação gemelar com gêmeo acárdio. Foram prescritas digoxina e corticoterapia. $\mathrm{Na} 29^{a}$ semana de gestação, o gêmeo 1 apresentou hepatomegalia e cardiomegalia, que se acentuaram na $31^{\text {a }}$ semana, quando se optou pela terapia invasiva. Após consentimento do casal, realizou-se embolização da artéria umbilical do gêmeo acárdio com álcool absoluto, com interrupção imediata do fluxo sanguíneo. O tempo total do procedimento foi de $10 \mathrm{mi}-$ nutos e não foi observado qualquer efeito na freqüência cardíaca ou no Doppler da artéria umbilical do gêmeo 1 . Novo controle realizado no dia seguinte ao procedimento confirmou a ausência de fluxo sangüíneo no gêmeo acárdio e fluxo umbilical normal no gêmeo 1. A gestação evoluiu com rotura de membranas 48 horas após o procedimento e nascimento de recém-nascido 1 masculino, Apgar 9/9, pesando 1.515 g. Sua evolução neonatal foi satisfatória, com regressão da hepatomegalia e cardiomegalia, recebendo alta hospitalar no $33^{\circ}$ dia de vida. O recém-nascido 2 correspondia ao gêmeo acárdio-acéfalo cujo exame anatomopatológico confirmou o diagnóstico (Figura 2). A placenta era monocoriônica-diamniótica e havia uma 
grande artéria trombosada na sua superfície entre a inserção dos cordões do gêmeo acárdio e do gêmeo normal. O cordão do gêmeo acárdio apresentava dois vasos e inserção velamentosa (Figura 3). Aos 2 anos de idade, a criança não apresenta seqüelas de desenvolvimento.

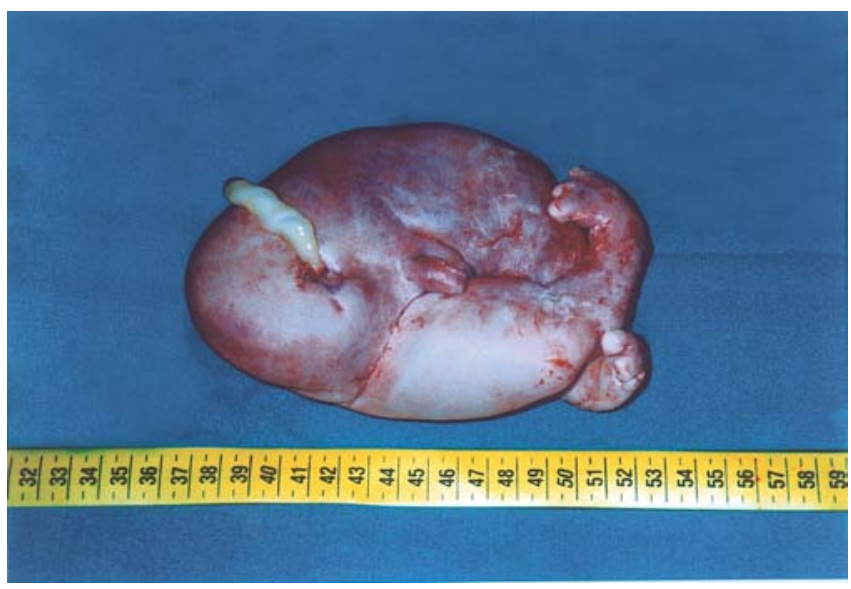

Figura 2 - Visão frontal do gêmeo acárdio constituído por massa de tecido hemorrágico pesando 400 gramas. Observa-se o bom desenvolvimento da genitália e dos membros inferiores.

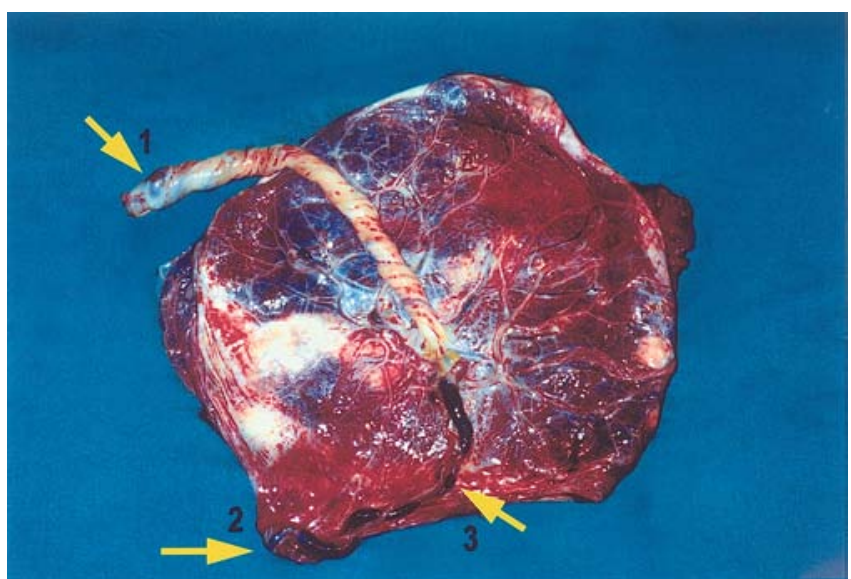

Figura 3 - Superfície da placenta do caso $n^{0} 2$. Observa-se a inserção marginal do cordão do gêmeo acárdio (2) e a fístula (3) entre os cordões do gêmeo bomba (1) e do gêmeo acárdio.

\section{Discussão}

Várias teorias têm sido propostas para explicar as alterações fisiopatológicas encontradas nas gestações com gêmeo acárdio ${ }^{5}$. A teoria vascular é a mais aceita e propõe o aparecimento precoce de anastomoses vasculares na placenta como defeito primário. Essas anastomoses implicariam uma perfusão contra-corrente de sangue arterial pobre em oxigênio para o feto com pressão arterial menor, resultando em hipóxia tecidual na fase de gênese de órgãos e membros fetais. Os efeitos da hipóxia são mais evidentes na metade superior do corpo, provavelmente por hipoperfusão, uma vez que necessita de maior pressão vascular para receber suprimento sangüíneo. Os órgãos irrigados pela aorta e artérias ilíacas são os que apresentam melhor desenvolvimento. Esta seqüência resulta em disrupção da morfogênese normal, levando a agenesia do crânio, membros superiores e órgãos torácicos ${ }^{5}$.

Há três mecanismos que podem explicar como o gêmeo acárdio afeta o bem-estar do gêmeo sadio, conhecido como gêmeo bomba. Primeiramente, o shunt sistêmico aumenta a demanda cardiovascular do gêmeo bomba, levando a insuficiência cardíaca e poliidrâmnio. Segundo, o crescimento contínuo do gêmeo acárdio, que normalmente supera o do gêmeo bomba, provocando distensão uterina, aumentando as complicações perinatais, particularmente parto prematuro. Terceiro, o sangue desoxigenado do gêmeo acárdio retorna ao gêmeo bomba pela anastomose placentária, reduzindo, dessa forma, o suprimento de oxigênio do gêmeo bomba, podendo levar a hipóxia crônica e restrição do crescimento ${ }^{2}$.

A taxa de mortalidade global do gêmeo bomba varia de 35 a 50\% $\%^{1}$. As complicações associadas a essa alta taxa de mortalidade incluem insuficiência cardíaca, poliidrâmnio e parto prematuro. O coração do gêmeo bomba perfunde os dois gêmeos e esse excesso de esforço leva à insuficiência cardíaca, traduzida por hipertrofia e dilatação em graus variados com estenose relativa da artéria pulmonar devido à hipertrofia ventricular direita. Hepatoesplenomegalia, ascite e poliidrâmnio são secundários à insuficiência cardíaca ${ }^{2}$.

Várias modalidades terapêuticas têm sido propostas, mas não há consenso sobre o melhor tipo de tratamento ${ }^{2,6}$. A utilização de método intervencionista apresenta melhores resultados em relação à conduta expectante, que se associa a alta mortalidade fetal ${ }^{3}$.

Os esforços terapêuticos iniciais tinham como objetivo o tratamento da insuficiência cardiaca utilizando digoxina ${ }^{7}$ e o controle do poliidrâmnio $^{8}$, inicialmente com indometacina e posteriormente por meio da amniodrenagem ${ }^{6}$.

A prescrição de medicamentos para o tratamento da síndrome TRAP foi utilizada a partir de 1983, porém seus resultados mostraram-se pouco promissores. A digitalização materna foi inicialmente proposta com o objetivo de tratar a insuficiência cardíaca fetal. No entanto, sua utilização não impede a evolução do quadro, como observou- 
se nos dois casos relatados, o que tem sugerido que se abandone essa modalidade terapêutica.

A administração de indometacina foi um dos métodos propostos para o controle do poliidrâmnio. No entanto, sua administração não impede a progressão da insuficiência cardíaca, podendo mesmo precipitá-la por fechamento prematuro do canal arterial. Além disso, não reduz a mortalidade perinatal e possui efeito potencialmente deletério sobre a função renal fetal.

Nossos casos confirmam a expectativa de alguns autores ${ }^{2,6}$ em relação à baixa eficácia do uso de drogas no controle de gestações com gêmeo acárdio. Por não atuarem sobre a fisiopatologia do problema, não impedem a evolução do quadro clínico, o que tem desestimulado sua utilização.

Amniodrenagem seriada foi o primeiro método invasivo proposto para o tratamento dessas gestações. Baseia-se no controle do poliidrâmnio, sendo necessária a repetição periódica do procedimento, aumentando o risco de complicações. Os benefícios dessa técnica incluem: aumento do fluxo sanguíneo nas artérias uterinas, demonstrado pelo estudo dopplerfluxométrico; diminuição da taxa de acúmulo de líquido amniótico e diminuição da compressão sobre a placenta, reduzindo a taxa de transfusão para o gêmeo receptor. As principais complicações observadas são: parto prematuro, rotura prematura de membranas, descolamento prematuro de placenta, corioamnionite e perda fetal.

A ineficácia dos métodos relatados em obter resultados perinatais satisfatórios levou ao desenvolvimento de técnicas com o objetivo de interromper a circulação entre o gêmeo acárdio e o gêmeo bomba, agindo sobre a fisiopatologia do problema. Várias técnicas foram descritas com esse objetivo, que são muito sedutoras, mas de realização complexa.

O primeiro caso de sucesso com o uso da terapia invasiva foi relatado em 1989 por Robie et al. ${ }^{9}$, que realizaram parto seletivo por histerotomia de um gêmeo acárdio com 22 semanas de gestação com subseqüente parto do gêmeo bomba saudável com 33 semanas, pesando 2.130 gramas.

Posteriormente, outras técnicas foram desenvolvidas e atualmente o tratamento dessas gestações utiliza dois métodos. O primeiro é a oclusão do cordão do gêmeo acárdio, guiada por ultra-som ou fetoscopia. É realizada de diferentes formas, incluindo ligadura do cordão por fetoscopia ${ }^{2}$, termocoagulação ${ }^{10}$, mini-histerotomia $^{2,9}$, coagulação a laser ${ }^{4} \mathrm{e}$ embolização com substâncias esclerosantes ${ }^{2,6,11}$. O outro método utilizado é a ablação intrafetal dos vasos pélvicos. O procedimento é guiado por ultra-som e utiliza, além de alguns métodos anteriores, a coagulação dos vasos fetais por radiofreqüência ${ }^{12}$.

Algumas dificuldades técnicas são observadas quando se utiliza a via funicular. Os cordões dos gêmeos acárdio e bomba estão intimamente situados e a justaposição da artéria e veia torna imperativa a identificação correta dos vasos do cordão umbilical do gêmeo acárdio durante sua oclusão. Além disso, o cordão umbilical do gêmeo acárdio normalmente é curto e estruturalmente anormal, propenso a acidentes secundários a ruptura ou sangramento. Por outro lado, a ablação intrafetal é tecnicamente mais fácil (possui baixa taxa de falha técnica), segura (apresenta menor incidência de rotura prematura de membranas e parto prematuro) e mais eficiente, quando comparada às técnicas de oclusão do cordão umbilical.

A técnica mais simples e mais utilizada para ablação intrafetal é a embolização com substâncias esclerosantes. O álcool absoluto é a substância mais utilizada com essa finalidade. Pelo seu efeito esclerosante, é largamente indicado na embolização de vasos arteriais em adultos e foi utilizado com sucesso pela primeira vez em gestações com feto acárdio por Holzgreve et al. ${ }^{13}$, em 1994. É procedimento relativamente simples que requer pouco equipamento, incluindo uma agulha de calibre 20 ou 22 e uma seringa de $5 \mathrm{~mL}$ contendo álcool absoluto. A técnica é semelhante à cordocentese, sendo de simples realização e com menor morbidade materna. A embolização é realizada na porção intra-abdominal da artéria umbilical do gêmeo acárdio, que é facilmente identificada por meio do Doppler, diminuindo os riscos de injeção intravenosa. Sua maior desvantagem, no entanto, é o risco de provocar apenas oclusão transitória, como observado no nosso primeiro relato, além da possibilidade de óbito do gêmeo bomba pela transferência da substância esclerosante para a sua circulação. Por isso, recomenda-se que essa técnica seja utilizada apenas em centros onde outras alternativas terapêuticas de ablação intrafetal não estejam disponíveis. As complicações ${ }^{11}$ relacionadas a esse procedimento incluem corioamnionite e rotura de membranas, como pôde ser observado em nosso segundo caso.

Nos últimos anos outras técnicas para ablação intrafetal foram desenvolvidas, utilizando diferentes fontes de energia. As mais difundidas são a termocoagulação ${ }^{10}$, o laser intersticial ${ }^{4}$ e a radiofreqüência ${ }^{12}$.

A termocoagulação é técnica simples e não requer equipamentos caros. Utiliza agulha de calibre $18 \mathrm{com}$ eletrodo de $1 \mathrm{~mm}$ de diâmetro conectado a um cautério e isolado com uma pro- 
teção de politetrafluoretileno cuja extremidade, que não excede $3 \mathrm{~mm}$, encontra-se descoberta. $\mathrm{O}$ procedimento pode ser realizado precocemente na gestação sob anestesia local e é facilmente reprodutivel. A termocoagulação oblitera todos os vasos umbilicais, levando à interrupção imediata do fluxo sangüíneo. Seu maior problema é o risco de dano ao tecido situado ao redor da cânula por coagulação direta ou por disseminação da corrente elétrica.

Outra técnica promissora é o laser intersticial. Embora os principios sejam semelhantes àqueles da termocoagulação, não há risco de cauterização inadvertida de outros tecidos. O período ideal para sua realização é até a $24^{\mathrm{a}}$ semana de gestação, uma vez que a eficácia do laser diminui com o crescimento dos vasos umbilicais ${ }^{4}$. Entretanto, poucos centros de medicina fetal têm acesso a essa técnica, devido ao alto custo e baixo uso do equipamento.

A técnica mais recente é a ablação por radiofreqüência, que utiliza uma agulha de calibre 14 e um gerador de radiofreqüência. É técnica que tem se mostrado eficaz no tratamento de uma variedade de situações clínicas, principalmente no tratamento de metástases hepáticas. É procedimento pouco invasivo que utiliza a técnica percutânea guiada por ultra-som, obliterando de forma eficaz o fluxo sanguíneo no gêmeo acárdio. A energia é aplicada apenas nos tecidos que estão em contato direto com a ponta da agulha, o que impede a lesão inadvertida de outras estruturas. Como inconvenientes, necessita de uma equipe formada por cirurgião, perinatologista e radiologista, e o procedimento deve ser realizado em bloco cirúrgico sob anestesia geral ou peridural. No entanto, por ser técnica recente, são necessários mais estudos para comprovar sua eficiência e segurança no tratamento dessas gestações.

Finalmente, a idade gestacional da intervenção permanece controversa, uma vez que metade dos casos não necessitará de tratamento, por interrupção espontânea do fluxo sangüíneo com parada de crescimento do gêmeo acárdio. O tratamento profilático precoce, quando indicado, pode ser realizado em todas as gestações com gêmeo acárdio em torno da $16^{\mathbf{a}}$ semana de gestação se há fluxo sangüíneo demonstrável pelo Doppler no gêmeo acárdio. Outra opção terapêutica é adotar conduta expectante e optar pelo tratamento invasivo somente quando se identifica descompensação hemodinâmica das condições fetais.

Acreditamos que o tratamento deve ser imperativo quando se evidencia deterioração hemodinâmica do gêmeo bomba, demonstrada em exames ultra-sonográficos seriados. No entanto, a opção entre tratamento profilático e acompanhamento ultra-sonográfico deve ser discutida com os pais. Se o tratamento é necessário, a via intrafetal de interrupção do suprimento vascular do gêmeo acárdio é vista como o tratamento de escolha, quando comparada às técnicas de oclusão do cordão umbilical. Em nosso meio, a utilização de substâncias esclerosantes para ablação intrafetal deve ser indicada nestas circunstâncias, por ser mais simples, segura, de fácil acesso e de menor custo.

\section{ABSTRACT}

Twin pregnancy with an acardiac twin is a rare event characterized by the presence of a placentary arterial anastomosis between the pump twin, morfologically normal, and the acardiac twin, causing a reverse circulation in one of the twins. The major complications are associated with cardiac failure in the normal twin, which is due to circulatory overload and prematurity. Many therapeutic options have been proposed, but there is no consensus about which one is the best therapy. However, intrafetal ablation of the umbilical artery of the acardiac fetus proved to be an inexpensive method that is easy to perform and highly efficient in controlling circulatory overload. We presented two cases of intra-fetal ablation and good evolution. A triplet pregnancy in which the intra-fetal ablation was done in a 29-week-old fetus that evolved to premature delivery of a healthy baby, and a twin pregnancy in which intra-fetal ablation was done in a 31-week-old fetus that evolved to premature rupture of membranes and the premature delivery of a healthy baby.

KEYWORDS: Twin pregnancy. Reversed arterial perfusion sequence. Acardiac twin.

\section{Agradecimentos}

Ao Prof. Mário Jorge Viegas pela realização dos exames de doplervelocimetria arterial e venoso do caso $n^{\circ}$ 1. Aos Drs. Heverton Pettersen e Marcos Faria pela contribuição no acompanhamento e tratamento do caso $n^{\circ} 1$.

\section{Referências}

1. Gembruch U, Viski S, Bagamery K, Berg C, Germer $\mathrm{U}$. Twin reversed arterial perfusion sequence in twin-to-twin transfusion syndrome after the death of the donor co-twin in the second trimester. Ultrasound Obstet Gynecol 2001; 17:153-6. 
2. Tan TY, Sepulveda W. Acardiac twin: a systematic review of minimally invasive treatment modalities. Ultrasound Obstet Gynecol 2003; 22:409-19.

3. Brassard M, Fouron JC, Leduc L, Grignon A, Proulx F. Prognostic markers in twin pregnancies with an acardiac fetus. Obstet Gynecol 1999; 94:409-14.

4. Arias F, Sunderji S, Gimpelson R, Colton E. Treatment of acardiac twinning. Obstet Gynecol 1998; 91:818-21.

5. Sogaard K, Skibsted L, Brocks V. Acardiac twins: pathophysiology, diagnosis, outcome and treatment. Six cases and review of the literature. Fetal Diagn Ther 1999; 14:53-9.

6. Simpson PC, Trudinger BJ, Walker A, Baird PJ. The intrauterine treatment of fetal cardiac failure in a twin pregnancy with an acardiac, acephalic monster. Am J Obstet Gynecol 1983; 147:842-4.

7. Jones JM, Sbarra AJ, Dililo L, Cetrulo CL, D’Alton ME. Indomethacin in severe twin-twin transfusion syndrome. Am J Perinatol 1993; 10:24-6.
8. Roberts D, Neilson JP, Weindling AM. Interventions for the treatment of twin-twin transfusion syndrome. Cochrane Database Syst Rev 2001; (1):CD002073.

9. Robie GF, Payne GG Jr, Morgan MA. Selective delivery of an acardiac, acephalic twin. N Engl J Med 1989; 320:512-3.

10.Sepulveda W, Corral E, Gutierrez J. A simple device for vascular occlusion of acardiac twins. Ultrasound Obstet Gynecol 2003; 21:386-8.

11.Sepulveda W, Bower S, Hassan J, Fisk NM. Ablation of acardiac twin by alcohol injection into the intra-abdominal umbilical artery. Obstet Gynecol 1995; 86:680-1.

12.Tsao K, Feldstein VA, Albanese CT, et al. Selective reduction of acardiac twin by radiofrequency ablation. Am J Obstet Gynecol 2002; 187:635-40.

13.Holzgreve W, Tercanli S, Krings W, Schuierer G. A simpler technique for umbilical-cord blockade of an acardiac twin. N Engl J Med 1994; 331:56-7.

\title{
XXVII Jornada de Ginecologia e Obstetrícia de Brasília
}

\section{9 de setembro a 01 de outubro de 2004}

\author{
Hotel Nacional - Brasília - DF \\ Informações: \\ SGOB \\ Tel.: (61) 245-3681 - Fax: (61) 245-4530 \\ e-mail: sgob@ambr.com.br
}

